\title{
Quantum point contact galvanically coupled to planar superconducting resonator for ultra-sensitive broad-band electrical amplification
}

\section{Prasanta Kumbhakar}

Indian Institute of Science Education and Research, Thiruvananthapuram

\section{Anusha Shanmugam}

Indian Institute of Science Education and Research, Thiruvananthapuram

\section{Chithra H Sharma}

Indian Institute of Science Education and Research, Thiruvananthapuram

\section{J. Reno}

Sandia National Labs

Madhu Thalakulam ( $\nabla$ madhu@iisertvm.ac.in )

Indian Institute of Science Education and Research, Thiruvananthapuram https://orcid.org/0000-00016805-9138

\section{Article}

Keywords: single charge dynamics, metrology

Posted Date: February 5th, 2021

DOI: https://doi.org/10.21203/rs.3.rs-145756/v1

License: (c) (1) This work is licensed under a Creative Commons Attribution 4.0 International License.

Read Full License 


\title{
Quantum point contact galvanically coupled to planar superconducting resonator for ultra-sensitive broad-band electrical amplification
}

\author{
${ }^{1}$ Prasanta Kumbhakar, ${ }^{1}$ Anusha Shanmugam, ${ }^{1}$ Chithra H Sharma, ${ }^{2}$ J. L. Reno, and ${ }^{1}$ Madhu Thalakulam* \\ ${ }^{1}$ Indian Institute of Science Education \& Research Thiruvananthapuram, Kerala, India 695551 \\ ${ }^{2}$ Center for Integrated Nanotechnologies, Sandia National Laboratory, Albuquerque, NM, USA
}

\begin{abstract}
Probing single charge dynamics in solids can give insights into various quantum transport phenomena, most of which are fragile and short-time-scaled. Detection of these events in realtime requires a mesoscopic electrical amplifier with unprecedented sensitivity and operational bandwidth. In this work, we explore a hybrid electrical amplifier consisting of a semiconducting quantum point contact galvanically coupled to a superconducting $\lambda / 2$ transmission-line resonator for ultra-fast and ultra-sensitive charge amplification. The resonator, made of Aluminium with a coplanar waveguide geometry, is designed to operate at its first harmonic resonant mode $\sim 3.4 \mathrm{GHz}$, where the reflected power from the resonator is amplitude-modulated by the conductance changes in the quantum point contact channel. From the sidebands of the amplitude modulated reflected signal we extract a conductance sensitivity of $2.85 \times 10^{-7}\left(e^{2} / h\right) / \sqrt{\mathrm{Hz}}(11.05 \mathrm{pS} / \sqrt{\mathrm{Hz}})$. This sensitivity translates to a unit signal-tonoise measurement time $\sim 1.62 \mathrm{~ns}$ for a variation of $0.01\left(e^{2} / h\right)$ in the conductance. The optimization of various operational parameters of the device reveals a bandwidth of $\sim 155 \mathrm{MHz}$ which corresponds to a rise-time $\sim 2.2 \mathrm{~ns}$. Both the sensitivity and bandwidth that we obtain are greater by an order compared to the existing reports. In addition, the device also exhibits very good sensitivities $\sim 3.58 \times 10^{-4}\left(e^{2} / h\right) / \sqrt{\mathrm{Hz}}$ up to a measurable frequency of $240 \mathrm{MHz}$. The extremely high sensitivity, ultra-fast operation reaching the nanosecond timescales, and the circuit QED architecture makes this scheme an attractive choice for single charge detection and counting experiments for spin-qubit readout and quantum electrical metrology.
\end{abstract}

\footnotetext{
*madhu@iisertvm.ac.in
} 


\section{Introduction}

The efforts to push the limits of precision and sensitivity of measurements have always been driven by the quest to probe and understand new phenomena, validate theoretical predictions, and conquer new technologies; for example, the detection of feeble signals of gravitational waves using laser-interferometric techniques nearly a century after its prediction ${ }^{1}$. Extremely weak signal levels combined with the fragile and ultra-fast nature make the detection of single electrons in solids a challenging task. Conventional transport measurements probe timeaveraged collective response of electrons while the key to understanding various quantum phenomena generated by many-body effects, electron-electron correlations, dephasing mechanisms in qubits, etc., require spatial and temporal access to individual charge carriers and the higher moments of their distribution ${ }^{2}$ which are not accessible during conventional transport measurements. Though, optical spectroscopy techniques can probe collective charge dynamics down to attosecond or even shorter timescales ${ }^{3}$, not sensitive to probe the spatial response of individual electrons. Time-resolved detection of single-electrons in solid circuits require a mesoscopic and ultra-sensitive broad-band charge/electrical amplifier with unprecedented spatial and temporal resolution.

The demand for charge sensors with better sensitivities and broader bandwidths is not only for capturing new physical phenomena but also is driven by developments in quantum enabled technologies such as solid-state quantum computing ${ }^{4}$ and, quantum electrical sensing and metrology 5 . The recent paradigmatic change of redefining the international system of units purely based on fundamental constants, the charge of the electron $e$, the Planks constant $h$ and, the speed of light in vacuum $c$ has a profound effect on the way the unit of current the Ampere is defined ${ }^{6}$. Realization of quantum current standard and the closure of the quantum electrical metrology triangle invariably involves measuring current in terms of the transfer rate of electrons in single-electron pumps which requires precise, ultra-fast, and highly sensitive charge detection and counting techniques. Ampere corresponds to an electron transfer rate of $6.24 \times 10^{18}$ (exa) $\mathrm{Hz}$, while the latest gated quantum dot based single-electron pumps operate in the $\mathrm{GHz}$ regime ${ }^{7}$, both of which are far beyond the reach of the current generation of singleelectron sensors ${ }^{8,9}$. The solid-state qubit technology has now reached a stage where the researchers are solving problems related to the interaction of the qubits with each other and the environment such as the de-coherence effects and the error-correction ${ }^{10}$. A major challenge in the practical implementation of quantum error correction protocols is that the state of the qubit needs to be determined via minimally invasive non-demolition measurements. The proposed 
ancilla-based repetitive quantum non-demolition measurement requires probing the qubits at rates a couple of orders faster than the dephasing rates of the states ${ }^{11,12}$. Though the fidelity of these measurements depends on the repetition rate, it has also been reported that the visibility contrast is strongly affected by the readout process ${ }^{13,14}$. All these points demand measurements at timescales much faster than the decoherence times and the requirement of a highly sensitive mesoscopic charge amplifier with large bandwidth.

Time-averaged single-charge detection has been accomplished using mesoscopic charge amplifiers, the single electron transistors $(\mathrm{SET})^{15}$ and the quantum point contacts $(\mathrm{QPC})^{16}$. Ever since the discovery of electrons, it took more than a century to detect its motion in realtime in solid ${ }^{17}$ where the single-electron charging-discharging events in a gated quantum dot are recorded using a radio-frequency superconducting single-electron transistor. Though sensitivities close to the theoretical limit have been reported for radio-frequency-SETs ${ }^{18,19}$ the operational bandwidth has been limited to a few tens of MHz. The ease of integration with semiconducting quantum circuits such as gated quantum dot qubits and fast single-electron pumps $^{7,20,21}$, and the operational simplicity makes QPCs a preferred choice over the SETs. Moreover, unlike the SETs which work on single-electron charging-discharging events imparting higher detector noise onto the system, QPCs are less noisy and have the potential to reach the quantum limit of detection ${ }^{22-24}$. Single-shot readout of electron tunnelling events in a quantum dot using an integrated QPC was achieved by the use of a cryogenic transconductance amplifier, but the bandwidth was limited to $\sim 100 \mathrm{kHz}^{4,21}$. Detection bandwidths $\sim 20 \mathrm{MHz}$ and charge sensitivities of $\sim 2 \times 10^{-4} e / \sqrt{\mathrm{Hz}}$ has been reported for radio-frequency QPCs ${ }^{9,25}$. Dispersive readout of charge configuration of a few-electron double quantum dot with a $10 \mathrm{MHz}$ bandwidth gate-sensor was also reported recently ${ }^{26}$. The inherent bandwidth of QPCs extends into the THz range ${ }^{27}$, nevertheless, uncontrollable circuit reactance owing to the lumped element nature of the RF tank circuit used in these experiments pauses serious limitation to the performance limiting the operation to sub-GHz frequencies with bandwidths $\sim$ a few $\mathrm{MHz}$ in frequencies.

In contrast, distributed element planar transmission-line resonators such as the coplanar waveguide $(\mathrm{CPW})$ resonators ${ }^{28,29}$ provide convenient alternatives while maintaining superior control over the operational parameters. Planar geometry also makes integration with other nanoscale devices such as gated quantum dots and superconducting circuits seamless. CPW resonators are widely used for circuit-QED applications in superconducting qubit circuits and 
lately have also been explored for coupling spin-states to the cavity modes in quantum-dot circuits $^{30-33}$. Recently, QPCs coupled to CPW resonators has been proposed for the study of electron-photon interaction ${ }^{34}$ and non-classical light driven photon assisted tunnelling in quantum conductors ${ }^{35}$.

In this work, we explore a hybrid device consisting of a semiconducting QPC galvanically coupled to a superconducting CPW resonator as an ultra-fast broadband mesoscopic electrical amplifier for various charge detection and counting applications. The resonator made of Aluminum (Al) is designed to operate $\sim 3.4 \mathrm{GHz}$ whose reflected power at the first-harmonic resonant mode is amplitude-modulated by the QPC conductance fluctuations. Conductance variations $\sim 0.002 e^{2} / h$ with a bandwidth $\sim 155 \mathrm{MHz}$ are captured from the resonator reflected power. Optimization of the operational parameters of the device, the QPC gatevoltage, RF power to the resonator, gate excitation frequency and amplitude reveals a sensitivity of $2.85 \times 10^{-7}\left(e^{2} / h\right) / \sqrt{\mathrm{Hz}}$ measured with an excitation frequency of $1.0 \mathrm{MHz}$. Our device also exhibits excellent sensitivity in the range of $\sim 10^{-4}\left(e^{2} / h\right) / \sqrt{\mathrm{Hz}}$ up to a signal frequency of $240 \mathrm{MHz}$. We note here that the operational frequency, bandwidth, and sensitivity achieved in this work are superior by an order to the reported values for similar sensors.

\section{Measurements and Results}

All measurements discussed in this manuscript are performed in a cryogen-free dilution refrigerator with a base temperature of $10 \mathrm{mK}$. The measurements utilize a radio-frequency reflectometry set-up as shown in Supplementary Information SI-1. The device consists of an Aluminium superconducting CPW resonator galvanically coupled to a QPC defined on a GaAs/AlGaAs two-dimensional electron gas $^{34,36}$ as shown in Fig. 1 (a) (top) while the equivalent circuit of the resonator-QPC system is shown in the bottom. We operate the resonator in its first-harmonic resonant mode. The DC and low-frequency source-drain voltages to the QPC have been introduced at the low-impedance points located at distances $\lambda / 4$ from both ends of the resonator using inductor-terminated CPW feedlines of length $\lambda / 2$. It has been shown elsewhere ${ }^{36}$, that this configuration allows the introduction of DC bias onto the central conductor without altering the first harmonic resonance characteristics, our operational regime of interest. Fig. 1 (b) shows an optical image of a representative Aluminium CPW resonator fabricated on a Sapphire substrate using standard micro-fabrication techniques. The inductors on the DC feed lines, shown in the upper inset, prevents the leakage of the RF-signal into the DC lines. We estimate an inductance $\sim 61.73 \mathrm{nH}$ for the inductor from the lithographic 

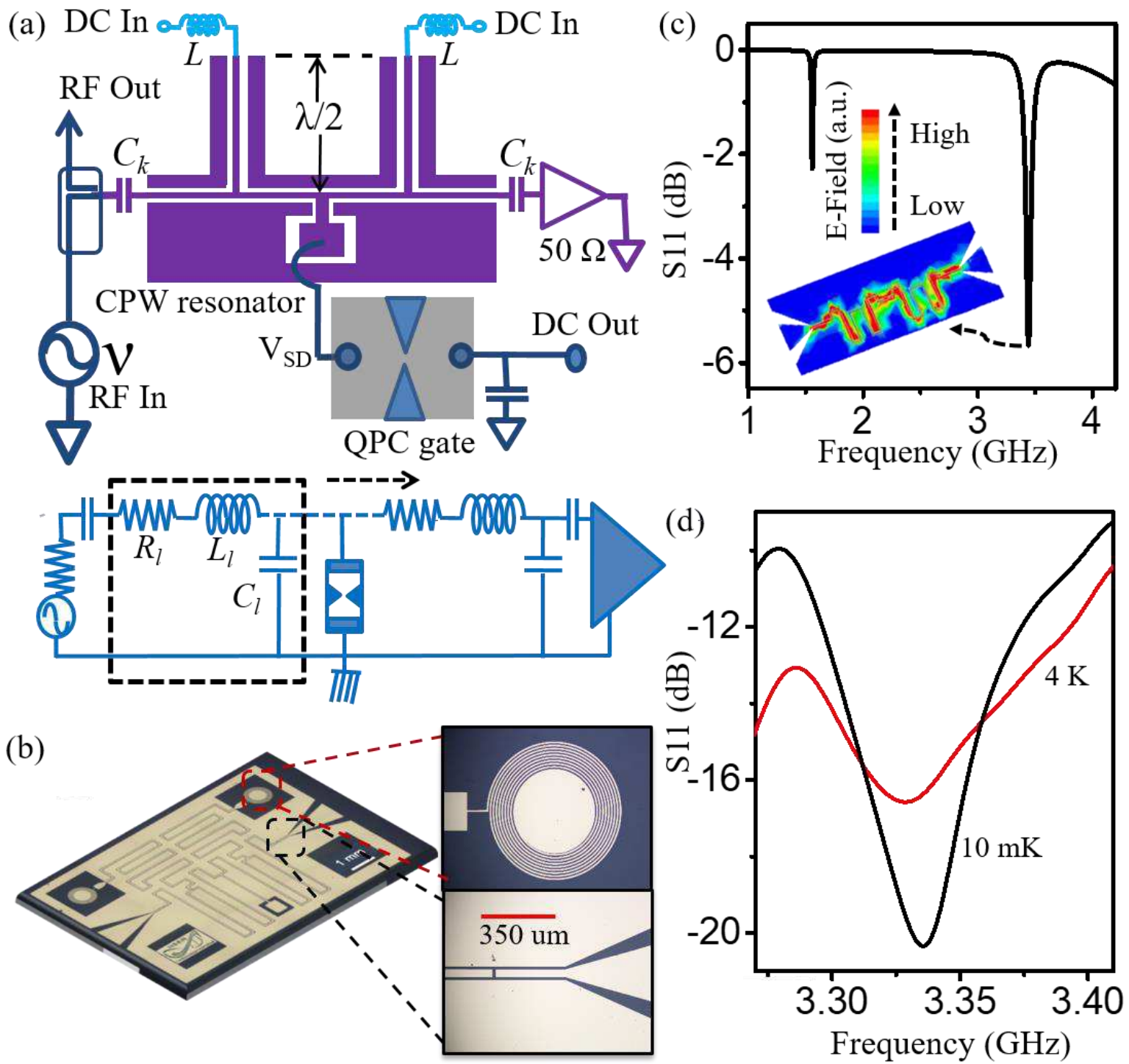

Fig.1 Measurement setup (a) Schematic diagram (top) and a circuit model(bottom) of the CPW resonator-QPC system. (b) Optical image of the CPW resonator and magnified views of the spiral inductor (top-right inset) and the coupling capacitor (bottom-right inset). (c) Simulated two-port reflectance S11 of the CPW resonator showing the fundamental and the $1^{\text {st }}$ harmonic resonant modes. Inset: The electric filed distribution for the $1^{\text {st }}$ harmonic mode $v_{1} \sim 3.4 \mathrm{GHz}$. (d) Measured S11 around $\mathrm{v}_{1} \sim 3.34 \mathrm{GHz}$ of the resonator-QPC system at $4 \mathrm{~K}$ (red) and $10 \mathrm{mK}$ (black).

dimensions ${ }^{37}$. The centre conductor of the resonator has a lithographic length $l \sim 3.7 \mathrm{~cm}$ and width $w \sim 100 \mu \mathrm{m}$. The separation between the centre conductor and the ground planes $s \sim 40 \mu \mathrm{m}$. The coupling capacitors to the main transmission line on either side are defined by gaps $\sim 25 \mu \mathrm{m}$ on the centre conductor [Fig. 1 (b) lower inset]. From the lithographic dimensions, we calculate an inductance per unit length $L_{l}=373.24 \mathrm{nH} / \mathrm{m}$, capacitance per unit length $C_{l}=169.03 \mathrm{pF} / \mathrm{m}$ and characteristic impedance, $Z_{0} \approx 47 \Omega^{29}$. We utilize computer simulations [ANSYS HFSS] to get further insight into the field distribution and frequency response of the resonator. Fig. 1 (c) shows the simulated two-port reflectance S11 
at the input port of a resonator with identical dimensions as the fabricated one, without the DC feed lines from which we obtain the fundamental $v_{0}$ and first harmonic $v_{1}$ resonant frequencies at $1.56 \mathrm{GHz}$ and $3.44 \mathrm{GHz}$ respectively. The inset shows the simulated electric field distribution for $v_{1}=3.44 \mathrm{GHz}$, our operating frequency, from which we confirm that the DC feed lines are introduced at locations of the minimum electric field. The coupling capacitor, $C_{k}$, calculated from the geometry $\sim 3.17 \mathrm{fF}$ puts the resonator-QPC system in the weak coupling regime with the external circuitry ${ }^{29,38}$. Fig. 1 (e) shows the measured S11 of the resonator-QPC system around $v_{1}=3.34 \mathrm{GHz}$ at $4 \mathrm{~K}(\mathrm{red})$ and $10 \mathrm{mK}$ (black). The measured resonant frequency $3.34 \mathrm{GHz}$ agrees within $2.3 \%$ of the simulated $v_{1}=3.44 \mathrm{GHz}$. The measured quality factor $Q_{L}$ at $10 \mathrm{mK}$ is $\sim 116$, while that at $4 \mathrm{~K}$ is $\sim 49$. From the relation, $1 / Q_{L}=$ $1 / Q_{\text {int }}+1 / Q_{\text {ext }}$ we obtain the internal quality factor ${ }^{29} Q_{\text {int }} \approx Q_{L}=116$ where, the external quality factor $Q_{\text {ext }} \sim 1.4 \times 10^{5}$. In this experiment, to enhance the resonator-QPC coupling, we directly connect the centre conductor to the source ohmic-contact to the QPC. We believe the dissipation and ohmic losses in the 2DEG and connecting wires could play a role in reducing the $Q_{i n t}{ }^{39}$. In support of this, we observe a reduction in the $Q_{L}$ from 116 to 49 as the resonator is taken from the superconducting state to the normal state [Fig. 1 (d)]. We also note here that the introduction of DC bias to the centre conductor via the $\lambda / 2$ feed lines does not affect the first-harmonic resonance of the cavity; refer Supplementary Information SI-2 for details.

The QPC is formed by the conventional Schottky split-gating technique ${ }^{40}$ on a GaAs/AlGaAs heterostructure (wafer no: VA 0286) hosting a two-dimensional electron gas (2DEG) $\sim 225 \mathrm{~nm}$ below the surface of the crystal, with a carrier concentration of $\sim 2.0217 \times 10^{11} \mathrm{~cm}^{-2}$ and a mobility of $\sim 2.13 \times 10^{6} \mathrm{~cm}^{2} \mathrm{~V}^{-1} \mathrm{~s}^{-1}$. Fig. 2 (a) shows the current through device vs. the gate voltage, the transconductance, of the QPC. The transconductance curve shows a complete pinch-off of the channel; a sharp drop in the current followed by a shutdown of the transport. The inset to Fig. 2 (a) shows a scanning electron microscope image of a QPC device with dimensions similar to the one studied in this manuscript. Fig. 2 (b) shows S11 vs. frequency for the resonator around $v_{1}=3.34 \mathrm{GHz}$ for various QPC gate-voltages corresponding to different conductance values along the pinch-off curve. As inferred from the experiment, the reflectivity of the resonator-QPC system at resonance is a strong function of the QPC conductance. We also observe that the $Q_{L}$ of the resonance at $v_{1}$ undergoes a substantial change, while the QPC channel is pinched-off, as shown in Fig. 2 (c). At resonance, the variation in the QPC conductance modulates the amplitude of the reflected RF signal. This 
aspect of the resonator-QPC system allowing one to capture the variations in the QPC resistance at timescales prescribed by the resonance-characteristics has important consequences in (i) probing ultra-fast charge transport across the device and (ii) charge readout process; CPW resonators being routinely used in circuit-QED applications makes this technique an attractive and compatible choice for qubit readout using QPC charge sensors. The rest of the studies described in this manuscript are based on the measurement and analysis of the amplitude-modulated reflected RF signal from the resonator-QPC system at resonance, $v_{1}$, unless otherwise specified. For this an input RF signal (carrier-wave) with frequency of $v_{1} \sim 3.34 \mathrm{GHz}$ with a power $\left(P_{\mathrm{rf}}\right)$ applied onto the resonator input port while a small AC signal, at a frequency $v_{m}$, inducing a small variation in the QPC conductance is applied to one of the
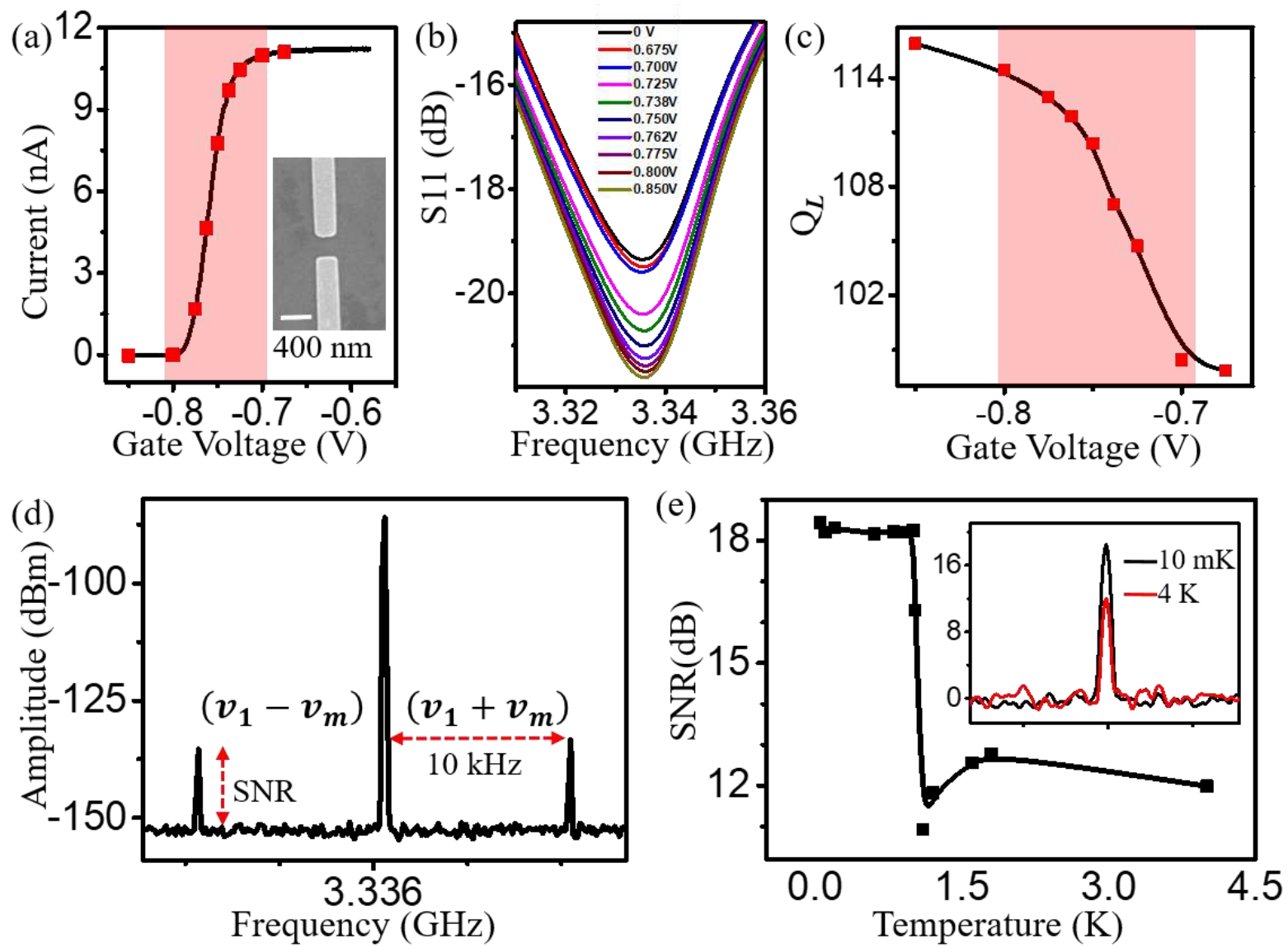

Fig.2. QPC transconductance and amplitude modulation. (a) QPC current vs. gate voltage taken at 10mK. Inset: SEM image of a QPC device similar to the measured one. (b) Reflectance S11 of the resonator-QPC system for various QPC conductance values, shown in red-squares in panel (a), along the pinch-off characteristics. (c) Qvalue of the resonator-QPC system as a function of QPC conductance extracted from the S11. (d) Reflected signal from the resonator-QPC system at a carrier frequency $v_{1} \sim 3.34 \mathrm{GHz}$ with a power $\sim-79 \mathrm{dBm}$ while an excitation signal of amplitude $1 \mathrm{mV}_{\mathrm{RMS}}$ at $10 \mathrm{kHz}$ applied onto one of the gates. The charge sensitivity is calculated from the SNR of the sidebands in the reflected power spectrum. (e) SNR vs. temperature with an excitation of $1 \mathrm{mV}$ rms at $10 \mathrm{kHz}$ applied to the QPC gate while an RF signal with a frequency $v_{1} \sim 3.34 \mathrm{GHz}$ with a power of $\sim-59 \mathrm{dBm}$ is applied to the resonator. Inset: a power spectrum of the $\left(v_{1}-v_{\mathrm{m}}\right)$ side-band at $4 \mathrm{~K}$ (red) and $10 \mathrm{mK}(\mathrm{black})$ showing a change of $7 \mathrm{~dB}$ while the resonator was taken across the superconducting transition temperature of Aluminium $\sim 1.2 \mathrm{~K}$. All SNR measurement performed at $100 \mathrm{~Hz}$ resolution band width. 
QPC gates in addition to the DC gate voltage. The power spectrum of the resulting amplitude modulated reflected signal is acquired with the help of an RF spectrum analyser as function of various device operating parameters.

First, we inspect the small-signal response of the device from which we extract the conductance sensitivity of our device. For this, we bias the QPC at a point along the steep portion of the pinch-off curve $\left(V_{\mathrm{g}}=-0.735 \mathrm{~V}\right)$ with a gate excitation signal $\sim 1 \mathrm{mV}_{\mathrm{RMS}}$ at $v_{m}=10 \mathrm{kHz}$ causing an RMS conductance oscillation $\Delta G=0.092 \mu \mathrm{S}\left(\sim 0.002 e^{2} / h\right)$ in the channel while maintaining $P_{\mathrm{rf}}=-69 \mathrm{dBm}$. Fig. 2 (d) shows a representative power spectrum of the reflected signal. The QPC conductance oscillation resulting from the gate excitation modulates the $Q_{\text {int }}$ at the frequency $v_{m}$ causing amplitude modulation of the reflected RF signal manifested as sidebands at frequencies $v_{1} \pm v_{m}$, as shown in Fig. 2 (d). From the SNR of these sidebands, one can extract the conductance sensitivity of the device $\delta G$ using the formula $\delta G=$ $(1 / 2) \Delta G(\mathrm{BW})^{-1 / 2} 10^{-\mathrm{SNR} / 20}$ where $\mathrm{BW}$ is the resolution bandwidth of the external RF circuitry $^{25}$. We extract a conductance sensitivity of $5.19 \times 10^{-6}\left(e^{2} / h\right) / \sqrt{\mathrm{Hz}}[0.2 \mathrm{nS} / \sqrt{\mathrm{Hz}}]$ from the data. We note here that the typical conductance changes experienced by QPC sensors against single-electron tunnelling events in nearby quantum dots are $\sim 0.01\left(e^{2} / h\right)$ or greater $^{9,21,25,41}$ in amplitude compared to which, the conductance oscillations we induce for these measurements are lower by an order in amplitude $9,25,26,42$.

Now we explore the role of the superconducting resonator in attaining a high SNR and sensitivity. Fig. 2 (e) shows the SNR as a function of the device temperature with a $10 \mathrm{kHz}$ $1 \mathrm{~m} V_{\mathrm{RMS}}$ gate excitation and $P_{\mathrm{rf}} \sim-59 \mathrm{dBm}$. As the sample temperature is increased from $10 \mathrm{mK}$ through $4 \mathrm{~K}$, the SNR shows little variation until a temperature of $\sim 1.1 \mathrm{~K}$ is reached beyond which the SNR drops sharply by $\sim 7 \mathrm{~dB}$. The inset to Fig. 2 (e) shows a power spectrum of the sidebands at $10 \mathrm{mK}$ (black) and $4 \mathrm{~K}$ (red) for reference. This temperature corresponds to the superconducting transition temperature for Aluminium ${ }^{43}$. The dissipation in the normal metal resonator results in a lower $Q_{\text {int }}$ above the transition temperature [as shown in Fig. 1 (d)] and consequently a reduced SNR.

Sensitivity and operational bandwidth are two crucial parameters, defining the merits and limits of a sensor. In this session, we discuss the optimization of the operational parameters, the QPC conductance, amplitude and frequency of the gate excitation and, carrier-wave power 
and frequency to demonstrate the high sensitivity and broadband amplification characteristics of the device. The QPC conductance and carrier wave frequency are optimized by inspecting the small-signal response of the device. We apply a gate excitation, $v_{m}=10 \mathrm{kHz}$, of amplitude $1 \mathrm{~m} V_{\mathrm{RMS}}\left(\sim 0.002 e^{2} / h\right)$ while maintaining $P_{\mathrm{rf}} \sim-69 \mathrm{dBm}$. Fig. 3 (a) shows the SNR vs. the QPC gate-voltage. The device exhibits high SNR while biased along the steeper region of the transconductance trace where the transport is dominated by tunnelling phenomena while, the SNR vanishes when biased along with the flatter regimes, where the channel is either completely open or closed and the contribution to transport via tunnelling is negligible. The shaded region is a guide to the eye representing the steeper regime of the transconductance curve shown in Fig. 2 (a). The resonator to QPC power coupling factor is given by $\lambda_{g}^{2}=\pi Z_{0} / R$ where $R$ is the dynamic QPC resistance and $Z_{0} \approx 50 \Omega$ is the characteristic impedance of the resonator $^{34}$. Fig. 3(b) shows the power coupled to the $\mathrm{QPC}, P_{\mathrm{QPC}}$, from the resonator as a function of QPC gate-voltage in the pinch-off region ${ }^{34,44}$. For amplitude modulated signals, the SNR of the side-bands is proportional to the carrier-wave input power, which in turn is proportional to the QPC conductance. Unlike the conventional lumped-element LCR circuitbased charge sensors ${ }^{9,26,45}$, which are impedance-matched to the external circuit in a narrow operational range, our device shows very good SNR for a wide range of gate-voltage in the pinch-off regime. Towards the lower-half of the pinch-off trace, the large conductance change for a given gate-excitation signal compensates the reduced power coupled to the QPC to provide an appreciable SNR. While, towards the upper-half of the pinch-off trace, though the transconductance trace gets less steep, the increase in the power coupled to the QPC provides a good SNR. The power coupled to the QPC and the sensitivity of the QPC transconductance plays a balancing act to provide a good SNR for an extended range of QPC operational voltage. This is beneficial especially in multiqubit charge readout schemes where gate compensations are needed to nullify the effects of parasitic coupling of various qubit gates on to the sensor to maintain a highly sensitive operating regime. Fig. 3 (c) shows the SNR vs the excitation amplitude taken with $v_{m}=10 \mathrm{kHz}$ and $P_{\mathrm{rf}} \sim-69 \mathrm{dBm}$. We observe that the SNR dependence on the excitation amplitude is consistent with the amplitude modulation picture. We note here that we are able to detect excitation signals as low as $0.2 \mathrm{~m} V_{\mathrm{RMS}}\left(\sim 0.0005 e^{2} / h\right)$ on the gate, an important aspect for an electrical amplifier aspiring to reach the quantum limit.

Fig. 3 (d) shows the left-sideband power, $P_{\mathrm{s}}$ as a function of $P_{\text {rf }}$ (black trace) while the calculated RF voltage across the $\mathrm{QPC}, V_{\mathrm{RF}}$, is shown in red. $V_{\mathrm{RF}}$ is given by $V_{\mathrm{RF}}=\sqrt{P_{\mathrm{QPC}} \times R}$, 
where $P_{\mathrm{QPC}}$ is the average power coupled to the QPC from the cavity and $R$ is the QPC resistance. $P_{\mathrm{s}}$ varies linearly with RF power as expected for amplitude-modulated signals.

We note here that we have limited the applied RF power and the resulting RF voltage across the QPC to lower values such that the heating effects and subsequent dissipation in the resonator are negligible. We also note here that the RF voltage-range used in this experiment is comparable to those used elsewhere ${ }^{9,25}$.

To investigate the bandwidth, first, we inspect the SNR as a function of carrier-wave frequency while maintaining a gate excitation of frequency and amplitude, $v_{m}=10 \mathrm{kHz}$ and $1 \mathrm{mV}$ RMS respectively and, $P_{\mathrm{rf}} \sim-69 \mathrm{dBm}$. Fig. 3 (e) shows a plot of the SNR as a function of carrier-wave frequency in the vicinity of the resonance. The SNR attains a maximum at the

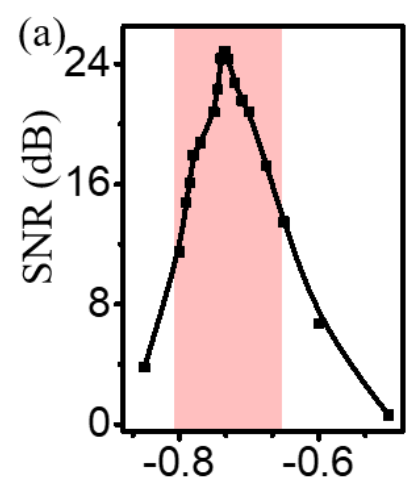

Gate Voltage (V)
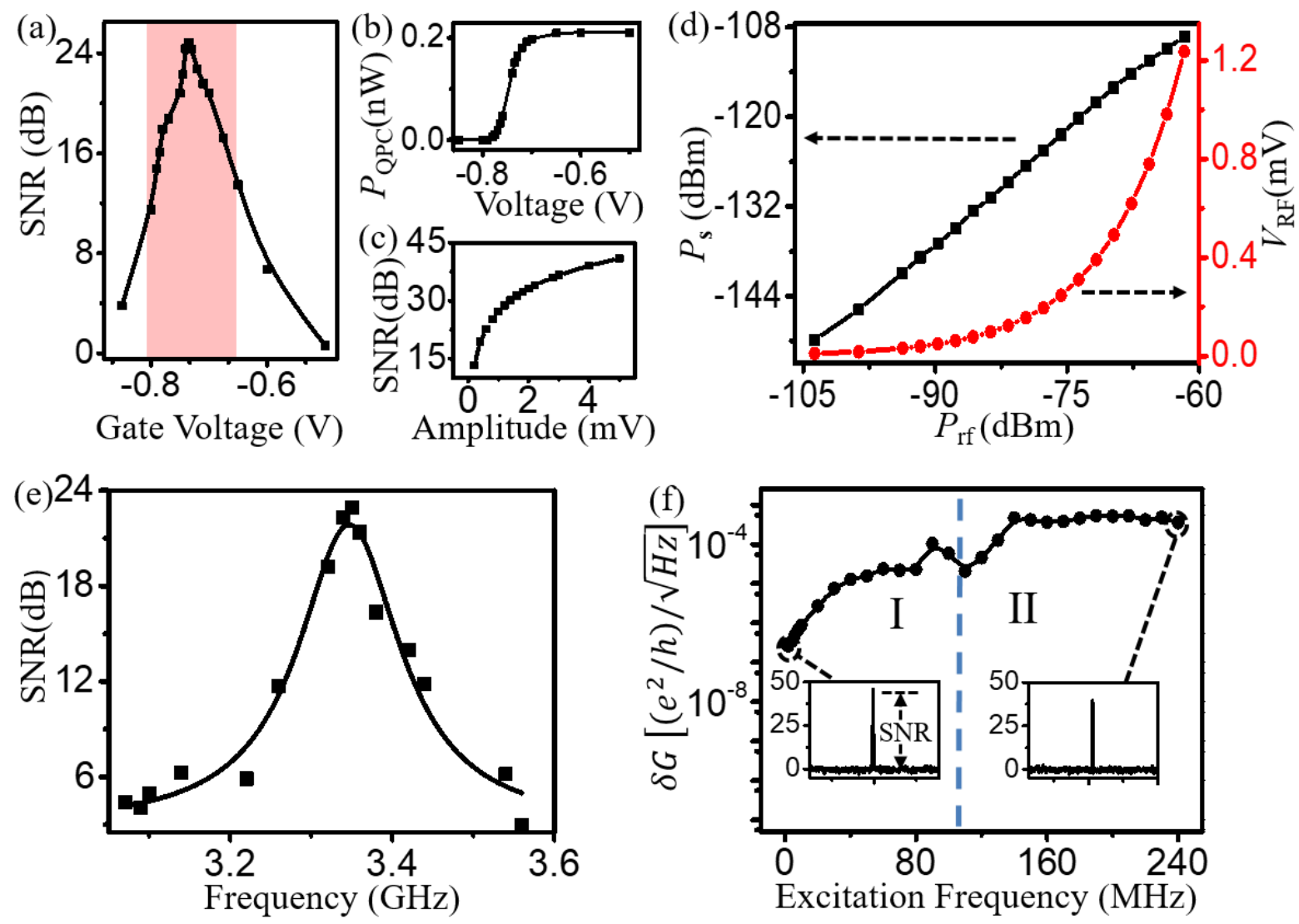

Fig.3. Broadband operation (a) SNR vs. the QPC gate voltage in response to a $P_{\mathrm{rf}} \sim-69 \mathrm{~dB}, v_{m}=10 \mathrm{KHz}$, $1 \mathrm{mV}_{\text {RMS. }}$ (b) RF power coupled to the QPC from the resonator vs. QPC gate-voltage. (c) SNR vs. excitation amplitude for $v_{m}=10 \mathrm{kHz}$ with $P_{\text {rf }} \sim-69 \mathrm{dBm}$. (d) SNR (left axis) vs. $P_{\text {rf }}$ with $v_{m}=10 \mathrm{kHz}$ with an amplitude of $1 \mathrm{mV}_{\mathrm{RMS}}$. The calculated RMS RF voltage across the QPC vs. the input power is shown in the right axis. (e) SNR vs. frequency of input RF signal. A bandwidth of $155 \mathrm{MHz}$ is extracted from the Lorentzian fit to the data. (f) Conductance sensitivity vs. excitation frequency for $P_{\mathrm{rf}} \sim-59 \mathrm{dBm}$ at $\sim 3.34 \mathrm{GHz}$ applied to the resonator. Insets: power spectrum of the sideband corresponding to $v_{m}=1 \mathrm{MHz}$ (left) and $240 \mathrm{MHz}$ (right) excitations. All the SNR measurements are performed with $100 \mathrm{~Hz}$ resolution band-width. 
resonant frequency, $v_{1} \sim 3.34 \mathrm{GHz}$ and falls-off as the frequency is varied to either side of the resonance. From a Lorentzian fit to the data we extract the bandwidth, the frequency span by which the SNR falls by $3 \mathrm{~dB}$ of the peak value, $\sim 155 \mathrm{MHz}$, corresponding to a rise-time of $\sim 2.2 \mathrm{~ns}$.

Next, we examine the broadband behaviour of our device by inspecting the sensitivity as a function of gate excitation frequency $v_{m}$. For this measurement, we maintain $P_{\mathrm{rf}} \sim-59 \mathrm{dBm}$ and apply an excitation $5 \mathrm{mV}$ at the input port of the homemade bias-tee. The amplitude of the signal reaching the QPC gate is dictated by the transmission vs. frequency (S21) of the biastee. Both the S21 and the amplitude of the gate excitation signal vs. frequency of the bias-tee are shown in the Supplementary Information SI-03. Fig. 3 (f) shows the conductance sensitivity as a function of $v_{m}$ calculated from the measured SNR. The device, though exhibit frequency dependence, shows excellent sensitivity up to $240 \mathrm{MHz}$ which correspond to response-times down to a few $n s$. The frequency dependence of the sensitivity, as shown in Fig. 3 (f), comes from two factors, (i) the S21 of the bias-tee. (ii) The bandwidth of the sensor, $\sim 155 \mathrm{MHz}$, shown in Fig. 3(e). Based on this we divide the Fig. 3 (f) into two regimes. In regime-I, the behaviour is mostly dictated by the bias-tee response, an artifact, while the bandwidth of the sensor is not the limiting factor. Though we apply a constant gate-excitation signal of $5 \mathrm{mV}_{\mathrm{RMS}}$ at the bias-tee input, the amplitude of the signal reaching the QPC gate increases with frequency [see Supplementary Information SI-03] resulting in a gradual reduction in the sensitivity. We note here that this observation is in concurrence with the data presented in Fig. 3 (c). In regimeII, the bandwidth of the resonator-QPC system dictates the sensitivity. The best sensitivity we obtain is $2.85 \times 10^{-7}\left(\mathrm{e}^{2} / \mathrm{h}\right) / \sqrt{\mathrm{Hz}}$ measured with an excitation frequency of $1.0 \mathrm{MHz}$, while we obtain sensitivities of $5.93 \times 10^{-5}\left(\mathrm{e}^{2} / \mathrm{h}\right) / \sqrt{\mathrm{Hz}}$ and $3.58 \times 10^{-4}\left(\mathrm{e}^{2} / \mathrm{h}\right) / \sqrt{\mathrm{Hz}}$ at $100 \mathrm{MHz}$, and $240 \mathrm{MHz}$ respectively.

\section{Conclusions}

Quantum point contacts are electrical amplifiers with inherent bandwidths extending into the $\mathrm{THz}$ regime ${ }^{27}$. The detection bandwidths and sensitivity has so far been limited to a few tens of $\mathrm{MHz}$, owing to the frequency-response characteristics of the associated electrical circuits. In this work, we realize a hybrid electrical amplifier consisting of a semiconducting QPC and a superconducting CPW resonator to achieve ultra-fast and ultra-sensitive detection. The QPC is galvanically coupled to the superconducting CPW resonator whose resonance characteristics are influenced by minute variations in the QPC conductance, enabling detection 
of these changes at frequencies and bandwidths dictated by the resonator. By this technique, we demonstrate a conductance sensitivity of $2.85 \times 10^{-7}\left(e^{2} / h\right) / \sqrt{\mathrm{Hz}}(\sim 11.02 \mathrm{pS} / \sqrt{\mathrm{Hz}})$ and an operational bandwidth of $\sim 155 \mathrm{MHz}$, which corresponds to a rise time of $\sim 2 \mathrm{~ns}$. The measurement time to detect a variation of $0.01\left(e^{2} / h\right)$ in the QPC conductance with unit SNR for our device is $\sim 1.6 \mathrm{~ns}$. Based on the available reports on quantum dot charge sensing using $\mathrm{QPCs}^{9,25}$, the conductance sensitivity we achieve translates to a charge sensitivity in the range of $\sim 10^{-6} \mathrm{e} / \sqrt{\mathrm{Hz}}$ range, referred to a capacitively coupled quantum dot. The device also exhibits excellent sensitivities of $\sim 3.58 \times 10^{-4}\left(e^{2} / h\right) / \sqrt{\mathrm{Hz}}$, up to $240 \mathrm{MHz}$ in bandwidth, which corresponds to a few nanoseconds in rise-time. Charge dynamics of duration nanoseconds or shorter are usually captured by optical spectroscopy techniques, but they are not sensitive down to individual electrons. The sensitivity and bandwidth we achieve enable detection of singlecharge dynamics down to a few nanoseconds in timescale adding plenty of room and power to comprehend various physical phenomena and develop new technologies where capturing single charge dynamics in real-time are central.

Methods: The QPC devices are fabricated using photo- and electron-beam lithography followed by metallization by physical vapour deposition technique. The Ohmic contacts to the $2 \mathrm{DEG}$ are realized using Indium alloying technique. The CPW resonator is fabricated on a sapphire substrate using photolithography followed by Aluminium metallization. The RF signal from the QPC drain is grounded to the resonator ground-plane using a $50 \mathrm{nF}$ SMD capacitor, while the DC and low frequency AC signal are return to the room-temperature for a direct measurement of the QPC current. All the electrical measurements are performed in a cryogen-free dilution refrigerator. Schematic diagram of the measurement setup is shown in Supplementary Information SI-01. The input RF signal to the resonator is attenuated at various temperature stages of the dilution refrigerator using coaxial inline attenuators. The reflected and the input signals are de-coupled using a directional-coupler (ZHDC-16-63$\mathrm{S}+$, Mini-Circuits) mounted at the $600 \mathrm{mK}$ stage. The reflected signal is further amplified by a $36 \mathrm{~dB}$ gain cryogenic HEMT amplifier (CITL F4, Cosmic Microwave Technology, Inc.) mounted at $4 \mathrm{~K}$ and a $9 \mathrm{~dB}$ gain room-temperature amplifier (ZJL-6G+, Mini-Circuits). Total attenuation of the input RF signal down to the resonator $\sim 56 \mathrm{~dB}$ while the total gain of the output line starting from the resonator to room temperature $\sim 42 \mathrm{~dB}$. $\mathrm{AC}$ gate excitation signals in the $\mathrm{kHz}$ range along with the DC voltage are applied using a summing amplifier, while in the $\mathrm{MHz}$ range with the help of home-made Bias-Tee whose frequency response is shown in Supplementary Information SI-03.

Acknowledgements: Authors acknowledge IISER Thiruvananthapuram for the facilities \& infrastructure. MT acknowledges the funding received from STARS under the project STARS/APR2019/PS/363. PK and CHS acknowledges Council of Scientific \& Industrial Research (CSIR), India for the fellowship. This work was performed, in part, at the Centre for Integrated Nanotechnologies, an Office of Science User Facility operated for the U.S. Department of Energy (DOE) Office of Science. Sandia National Laboratories is a multimission laboratory managed and operated by National Technology \& Engineering Solutions of Sandia, LLC, a wholly owned subsidiary of Honeywell International, Inc., for the U.S. DOE's National Nuclear Security Administration under contract DE-NA-0003525. The views expressed in the article do not necessarily represent the views of the U.S. DOE or the United States Government.

Author Contributions: MT conceived the problem. JLR grew the quantum well heterostructure wafers, PK designed and fabricated the devices. PK, AS and CHS did the experiments, PK analysed the data and, MT and PK co-wrote the manuscript. 


\section{References}

1. B. P. Abbott et al. Observation of gravitational waves from a binary black hole merger. Phys. Rev. Lett. 116, 1-16 (2016).

2. LEVITOV, L. \& LESOVIK, G. Charge distribution in quantum shot noise. JETP letters (1993) 58(3) 230-235 vol. 58 230-235 (1993).

3. Ramasesha, K., Leone, S. R. \& Neumark, D. M. Real-Time Probing of Electron Dynamics Using Attosecond Time-Resolved Spectroscopy. Annu. Rev. Phys. Chem. 67, 41-63 (2016).

4. J. M. Elzerman, R. Hanson, L. H. Willems van Beveren, B. Witkamp, L. M. K. V. \& L. P. K. $\&$ Kavli. Single-shot read-out of an individual electron spin in a quantum dot. $|V O L 430| 22$ JULY 2004 | www.nature.com/natur 430, 431-435 (2004).

5. Scherer, H. \& Camarota, B. Quantum metrology triangle experiments: A status review. Meas. Sci. Technol. 23, (2012).

6. Stock, M., Davis, R., De Mirandés, E. \& Milton, M. J. T. Corrigendum: The revision of the SI - The result of three decades of progress in metrology (Metrologia (2019) 56 (022001) DOI: 10.1088/1681-7575/ab0013). 2019 Metrol. 56022001 56, (2019).

7. Giblin, S. P. et al. Towards a quantum representation of the ampere using single electron pumps. Nat. Commun. 3, (2012).

8. Xue, W. W. et al. On-chip matching networks for radio-frequency single-electron transistors. Appl. Phys. Lett. 91, 9-12 (2007).

9. Cassidy, M. C. et al. Single shot charge detection using a radio-frequency quantum point contact. Appl. Phys. Lett. 91, 1-4 (2007).

10. Vandersypen, L. M. K. \& Eriksson, M. A. Quantum computing with semiconductor spins. Phys. Today 72, 38-45 (2019).

11. Nakajima, T. et al. Quantum non-demolition measurement of an electron spin qubit. Nat. Nanotechnol. 14, 555-560 (2019).

12. Xue, X. et al. Repetitive Quantum Nondemolition Measurement and Soft Decoding of a Silicon Spin Qubit. Phys. Rev. X 10, 21006 (2020).

13. Meier, F. \& Loss, D. Reduced visibility of Rabi oscillations in superconducting qubits. Phys. Rev. B - Condens. Matter Mater. Phys. 71, 1-5 (2005).

14. Vion, D., Aassime, A., Cottet, A., Joyez, P. \& Pothier, H. Manipulating the Quantum State of an Electrical Circuit. 296, 886-889 (2002).

15. M Field et al. Non-invasive detection of single-electron processes. Semicond. Sci. Technol. 11 1498-1501 (1996).

16. Willems van Beveren, L. H. et al. Few-electron quantum dot circuit with integrated charge read out. Phys. Rev. B - Condens. Matter Mater. Phys. 67, 9-12 (2003).

17. Lu, W., Ji, Z., Pfeiffer, L., West, K. W. \& Rimberg, A. J. Real-time detection of electron 
tunnelling in a quantum dot. Nature 423, 422-425 (2003).

18. Brenning, H., Kafanov, S., Duty, T., Kubatkin, S. \& Delsing, P. An ultrasensitive radiofrequency single-electron transistor working up to 4.2 K. J. Appl. Phys. 100, (2006).

19. Xue, W. W. et al. Measurement of quantum noise in a single-electron transistor near the quantum limit. Nat. Phys. 5, 660-664 (2009).

20. Hornibrook, J. M. et al. Frequency multiplexing for readout of spin qubits. Appl. Phys. Lett. 104, 1-5 (2014).

21. Vandersypen, L. M. K. et al. Real-time detection of single-electron tunneling using a quantum point contact. Appl. Phys. Lett. 85, 4394-4396 (2004).

22. Devoret, M. H. \& Schoelkopf, R. J. Amplifying quantum signals with the single-electron transistor. Nature 406, 1039-1046 (2000).

23. Turek, B. A. et al. Single-electron transistor backaction on the single-electron box. Phys. Rev. B - Condens. Matter Mater. Phys. 71, 2-5 (2005).

24. Rimberg, A. J. et al. The quantum limit for electrical amplifiers: Can we reach it? MEMS/MOEMS Components Their Appl. V. Spec. Focus Top. Transducers Micro-Nano Interface 6885, 688505 (2008).

25. Reilly, D. J., Marcus, C. M., Hanson, M. P. \& Gossard, A. C. Fast single-charge sensing with a rf quantum point contact. Appl. Phys. Lett. 91, 1-4 (2007).

26. Colless, J. I. et al. Dispersive readout of a few-electron double quantum dot with fast rf gate sensors. Phys. Rev. Lett. 110, 1-5 (2013).

27. Song, J. W. et al. Terahertz response of quantum point contacts. Appl. Phys. Lett. 92, 6-9 (2008).

28. Wallraff, A. et al. Strong coupling of a single photon to a superconducting qubit using circuit quantum electrodynamics. Nature 431, 162-167 (2004).

29. Göppl, M. et al. Coplanar waveguide resonators for circuit quantum electrodynamics. J. Appl. Phys. 104, 1-8 (2008).

30. Frey, T. et al. Dipole coupling of a double quantum dot to a microwave eesonator. Phys. Rev. Lett. 108, 1-5 (2012).

31. Mi, X. et al. A coherent spin-photon interface in silicon. Nature 555, 599-603 (2018).

32. Samkharadze, N. et al. Strong spin-photon coupling in silicon. Science (80-. ). 359, 1123-1127 (2018).

33. Scarlino, P. et al. Coherent microwave-photon-mediated coupling between a semiconductor and a superconducting qubit. Nat. Commun. 10, (2019).

34. Mendes, U. C. \& Mora, C. Electron-photon interaction in a quantum point contact coupled to a microwave resonator. Phys. Rev. B 93, 1-16 (2016).

35. Souquet, J. R., Woolley, M. J., Gabelli, J., Simon, P. \& Clerk, A. A. Photon-assisted tunnelling with nonclassical light. Nat. Commun. 5, (2014). 
36. Chen, F., Sirois, A. J., Simmonds, R. W. \& Rimberg, A. J. Introduction of a dc bias into a high- Q superconducting microwave cavity. Appl. Phys. Lett. 98, 19-22 (2011).

37. Mohan, S. S., Hershenson, M. D. M., Boyd, S. P. \& Lee, T. H. Simple accurate expressions for planar spiral inductances. IEEE J. Solid-State Circuits 34, 1419-1420 (1999).

38. Dib, N. Comprehensive study of CAD models of several coplanar waveguide (CPW) discontinuities. IEE Proc. Microwaves, Antennas Propag. 152, 69-76 (2005).

39. Petersson, K. D. et al. Circuit quantum electrodynamics with a spin qubit. Nature 490, 380383 (2012).

40. van Houten, H., Beenakker, C. W. J. \& van Wees, B. J. Quantum Point Contacts. Semicond. Semimetals 35, 9-112 (1992).

41. Müller, T. et al. An in situ tunable radio-frequency quantum point contact. Appl. Phys. Lett. 97, 1-4 (2010).

42. Vink, I. T., Nooitgedagt, T., Schouten, R. N., Vandersypen, L. M. K. \& Wegscheider, W. Cryogenic amplifier for fast real-time detection of single-electron tunneling. Appl. Phys. Lett. 91, (2007).

43. J. F. COCHRAN, D. E. MAPOTHRR, A. \& MOULDY, R. E. Superconducting Transition in Aluminum. PRB 103, (1956).

44. Müller, T. et al. A circuit analysis of an in situ tunable radio-frequency quantum point contact. Rev. Sci. Instrum. 84, (2013).

45. Schoelkopf, R. J., Wahlgren, P., Kozhevnikov, A. A., Delsing, P. \& Prober, D. E. The radiofrequency single-electron transistor (RF-SET): A fast and ultrasensitive electrometer. Science (80-. ). 280, 1238-1242 (1998). 
(a)

(a) DC In o
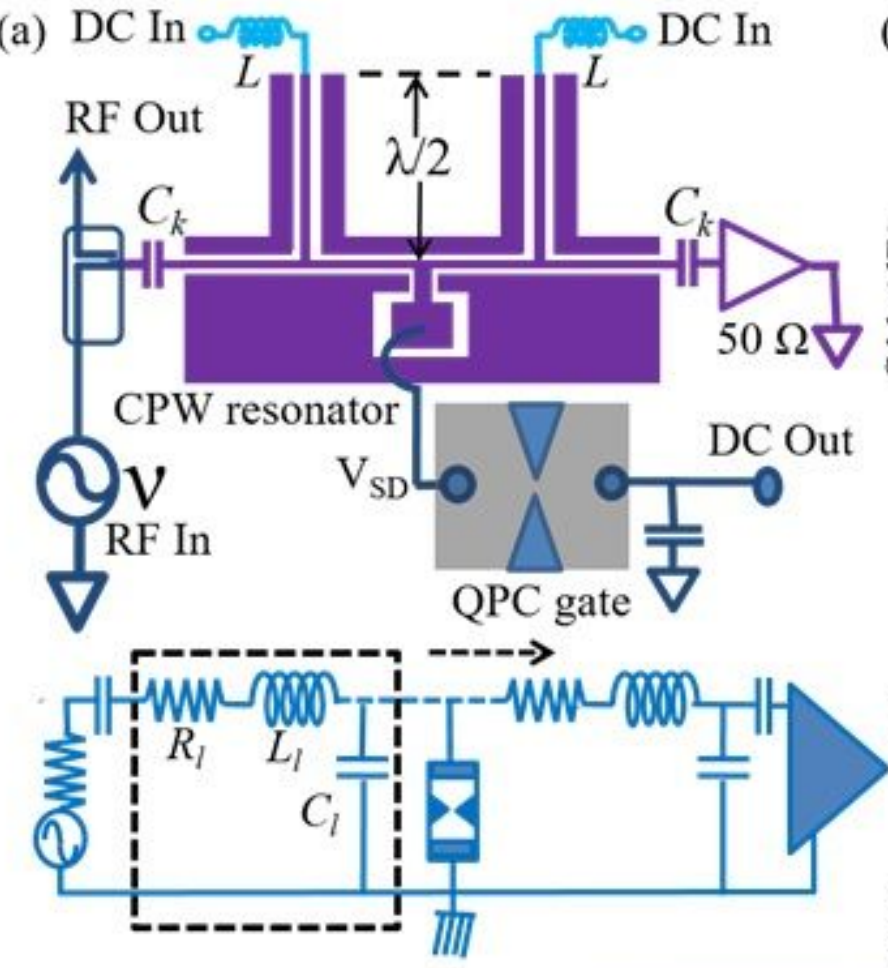

(b)

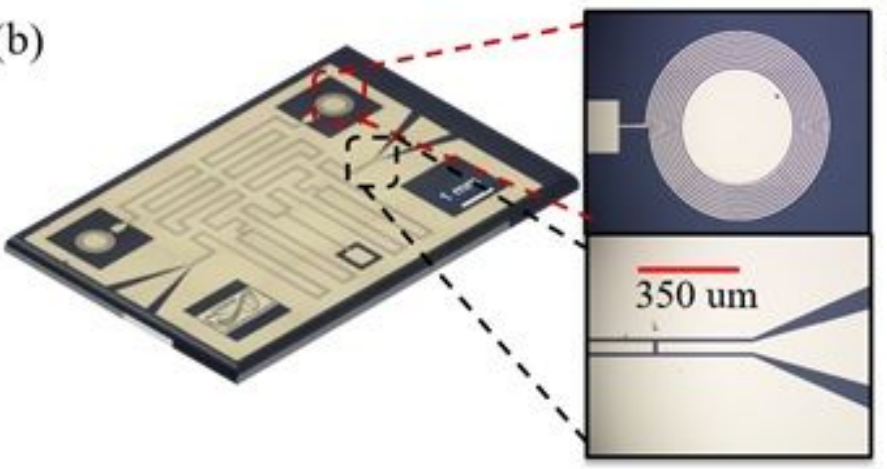

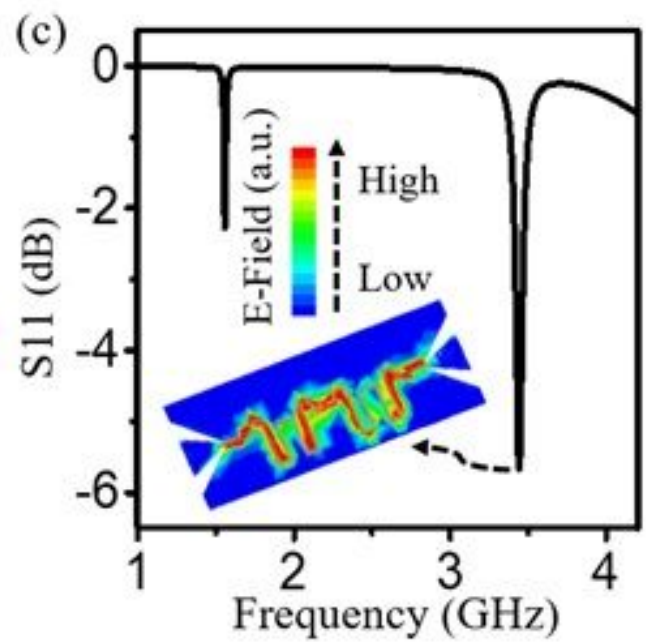

(d)

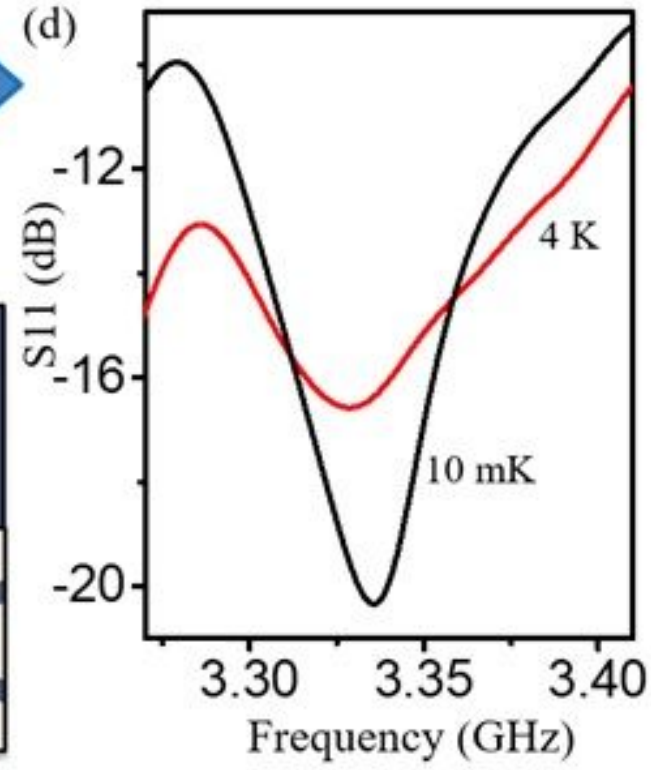

Figure 1

Measurement setup (a) Schematic diagram (top) and a circuit model(bottom) of the CPW resonator-QPC system. (b) Optical image of the CPW resonator and magnified views of the spiral inductor (top-right inset) and the coupling capacitor (bottom-right inset). (c) Simulated two-port reflectance S11 of the CPW resonator showing the fundamental and the 1 st harmonic resonant modes. Inset: The electric filed distribution for the 1st harmonic mode v_1 3.4 GHz. (d) Measured S11 around v_1 3.34 GHz of the resonator-QPC system at $4 \mathrm{~K}$ (red) and $10 \mathrm{mK}$ (black). 


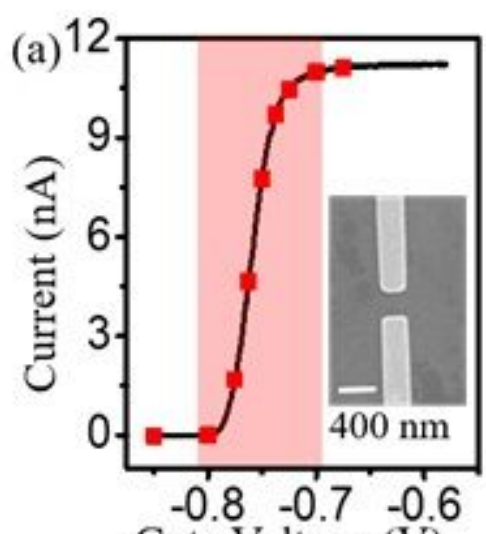

Gate Voltage (V)

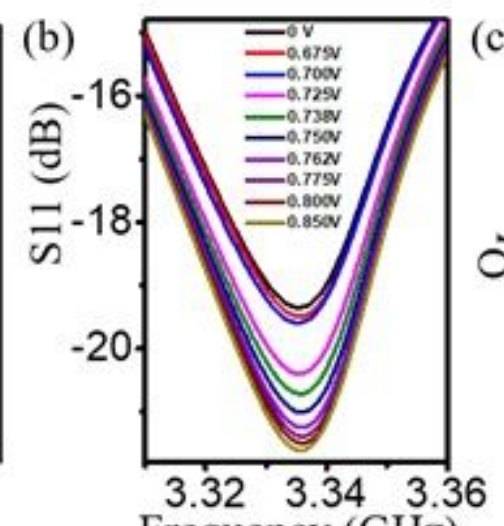

Frequency $(\mathrm{GHz})$

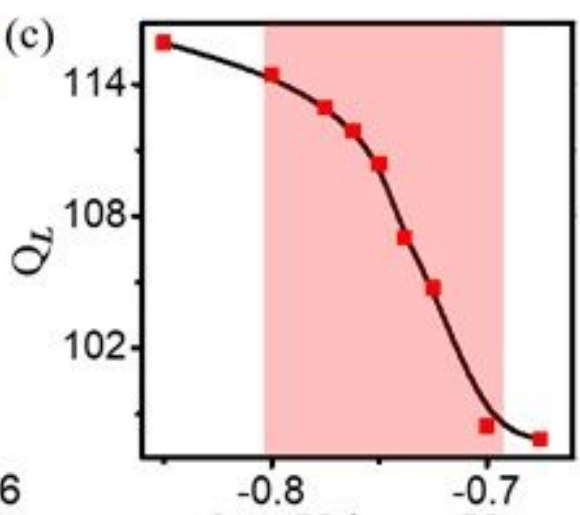

Gate Voltage (V)
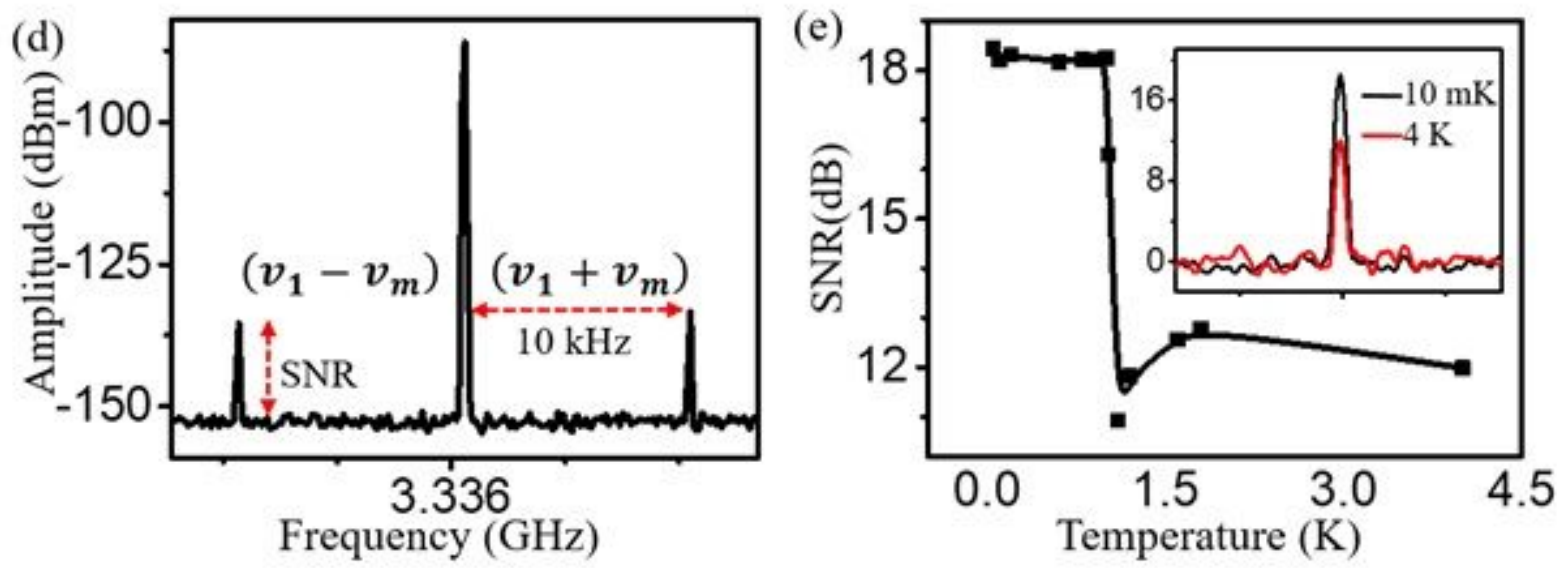

Figure 2

QPC transconductance and amplitude modulation. (a) QPC current vs. gate voltage taken at $10 \mathrm{mK}$. Inset: SEM image of a QPC device similar to the measured one. (b) Reflectance S11 of the resonator-QPC system for various QPC conductance values, shown in red-squares in panel (a), along the pinch-off characteristics. (c) Q-value of the resonator-QPC system as a function of QPC conductance extracted from the S11. (d) Reflected signal from the resonator-QPC system at a carrier frequency $₫ 1 \sim 3.34 \mathrm{GHz}$ with a power $\sim-79 \mathrm{dBm}$ while an excitation signal of amplitude $1 \mathrm{mVRMS}$ at $10 \mathrm{kHz}$ applied onto one of the gates. The charge sensitivity is calculated from the SNR of the sidebands in the reflected power spectrum. (e) SNR vs. temperature with an excitation of $1 \mathrm{mV}$ rms at $10 \mathrm{kHz}$ applied to the QPC gate while an RF signal with a frequency $\nabla 1 \sim 3.34 \mathrm{GHz}$ with a power of $\sim-59 \mathrm{dBm}$ is applied to the resonator. Inset: a power spectrum of the $(\nabla 1-\nabla \mathrm{m})$ side-band at $4 \mathrm{~K}$ (red) and $10 \mathrm{mK}$ (black) showing a change of $7 \mathrm{~dB}$ while the resonator was taken across the superconducting transition temperature of Aluminium $\sim 1.2 \mathrm{~K}$. All SNR measurement performed at $100 \mathrm{~Hz}$ resolution band width. 


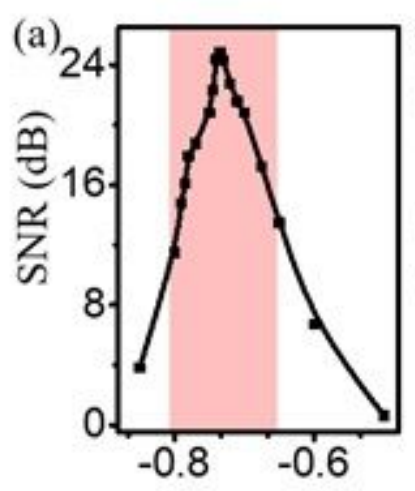

Gate Voltage (V)

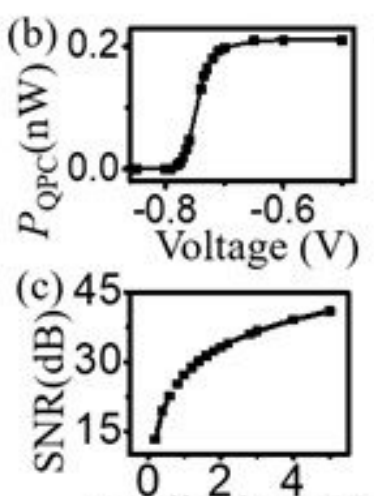

Amplitude (mV)
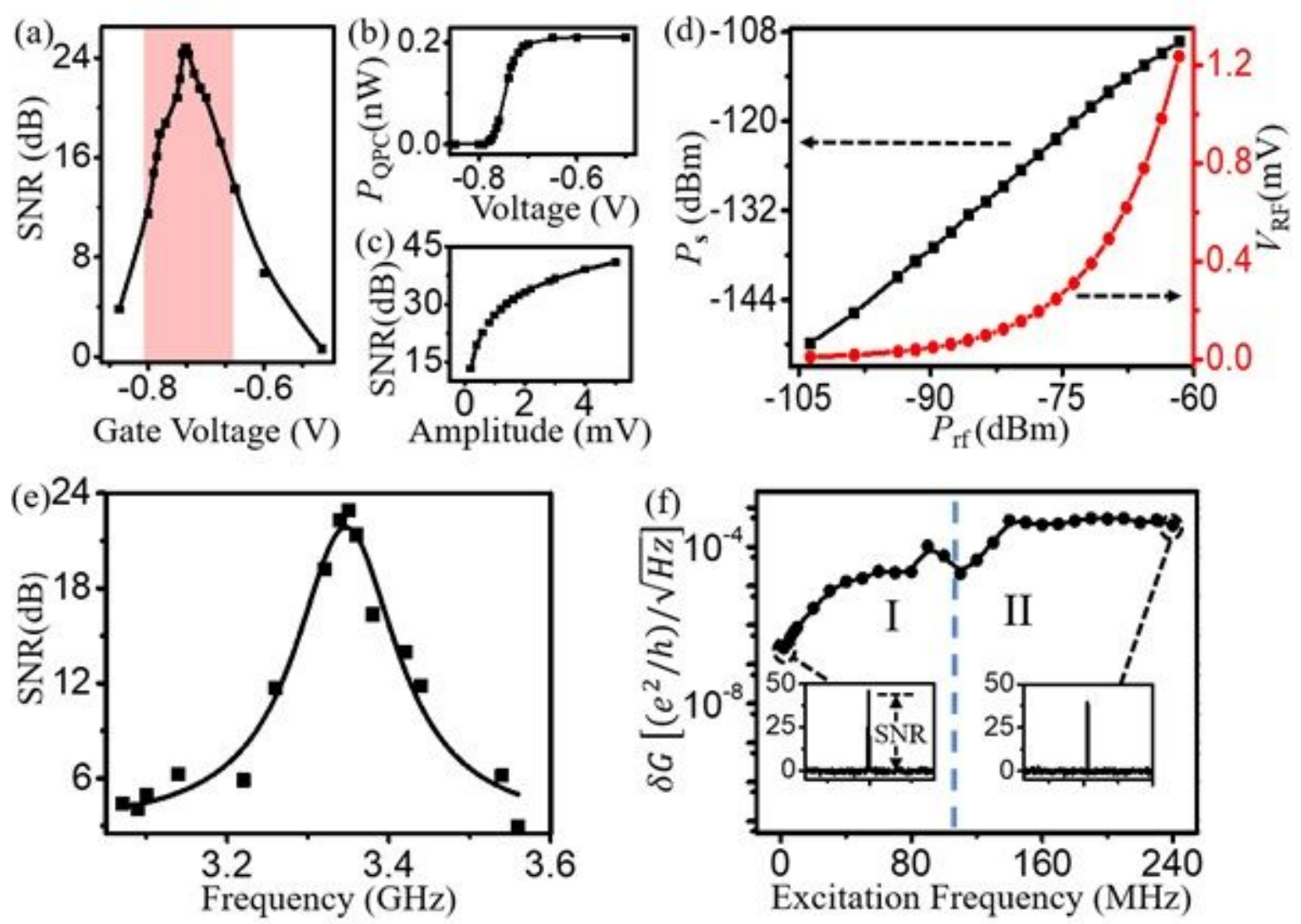

Figure 3

Broadband operation (a) SNR vs. the QPC gate voltage in response to a Prf - $69 \mathrm{~dB}, \mathrm{v} \_\mathrm{m}=10 \mathrm{KHz}$, 1mVRMS. (b) RF power coupled to the QPC from the resonator vs. QPC gate-voltage. (c) SNR vs. excitation amplitude for v_m $=10 \mathrm{kHz}$ with Prf $\sim-69 \mathrm{dBm}$. (d) SNR (left axis) vs. Prf with v_m=10 kHz with an amplitude of $1 \mathrm{mVRMS}$. The calculated RMS RF voltage across the QPC vs. the input power is shown in the right axis. (e) SNR vs. frequency of input RF signal. A bandwidth of $155 \mathrm{MHz}$ is extracted from the Lorentzian fit to the data. (f) Conductance sensitivity vs. excitation frequency for Prf $\sim-59 \mathrm{dBm}$ at $~ 3.34 \mathrm{GHz}$ applied to the resonator. Insets: power spectrum of the sideband corresponding to $\mathrm{v} \_\mathrm{m}=1$ $\mathrm{MHz}$ (left) and $240 \mathrm{MHz}$ (right) excitations. All the SNR measurements are performed with $100 \mathrm{~Hz}$ resolution band-width.

\section{Supplementary Files}

This is a list of supplementary files associated with this preprint. Click to download.

- Slv9.pdf 